\title{
Cloning, expression and purification of fructosyl amino acid oxidase (FAOX) in Escherichia Coli
}

\author{
Ho Ta Giap ${ }^{1 *}$, Phan Ngoc Han ${ }^{1}$, Tran Le Duy Phuong ${ }^{1}$, Phung Thi Thu Huong ${ }^{1}$, Vu Van Van ${ }^{1}$ \\ ${ }^{1}$ Nguyen Tat Thanh University, Vietnam \\ *Corresponding author: htgiap@ntt.edu.vn
}

ARTICLE INFO

DOI: 10.46223/HCMCOUJS. tech.en.11.1.1238.2021

Received: October $27^{\text {th }}, 2020$

Revised: January $13^{\text {rd }}, 2021$

Accepted: January $26^{\text {th }}, 2021$

Keywords:

fructosyl amino acid oxidase, FAOX-TE, expression and purification
Introduction: The level of serum $\mathrm{HbA} 1 \mathrm{c}$ is an indicator of the average blood sugar level in the last three months. HbA1c can be quantified using assays involving the enzyme fructosyl amino acid oxidase (FAOX). This study aims to produce GST-tagged FAOXTE (GST/FAOX-TE), a thermal stable and specific variant of FAOX, for future application studies.

Materials and methods: The E. coli strains DH5 $\alpha$ and BL21 (DE3) were used as cloning and expression hosts, respectively. The FAOX-TE sequence was synthesized at IDT (US) and clonned into pGEX-4T3 vector, which was confirmed by Colony PCR. The expression was induced at $16{ }^{\circ} \mathrm{C}, 0.5 \mathrm{mM}$ IPTG in LB media containing $50 \mu \mathrm{g} / \mathrm{ml}$ ampicilin. The protein expression profile was analyzed by SDS-PAGE. The cell pellet was sonicated and purified by Glutathione Sepharose 4 Fast Flow (Cytiva, US). The catalytic activity of GST/FAOX-TE with fructosyl valine was determined using high performance anion exchange chromatography with pulsed amperometry detection (HPAEC-PAD).

Results: The fusion protein was successfully expressed in Escherichia coli using the plasmid pGEX-4T3 and purified to high purity 93\%. Recombinant GST/FAOX-TE was shown to be active on fructosyl valine.

Conclusions: Active GST/FAOX-TE was successfully expressed in E. coli BL21 (DE3) and purified, which will be used for future development of biosensors for fructosyl valine quantification.

\section{Introduction}

The excess glucose in the bloodstream is the cause of the glycation of proteins, including hemoglobin, via non-enzymatic reactions (Bunn, Haney, Kamin, Gabbay, \& Gallop, 1976). Glycated hemoglobin (HbA1c) is thus an important marker for diabetes (Bunn et al., 1976). The HbA1c level in blood indicates the average blood glucose level in the last three months, which is an important factor to assess the severity of diabetes in patients (Ferri, Kim, Tsugawa, \& Sode, 2009). The $N$-terminal valine residue of $\mathrm{HbA1c}$ is glycated to form d-fructosyl-1-valine (FV). In clinical laboratories, HbA1c level can be quantified using different method, such as highperformance liquid chromatography (HPLC), affinity chromatography, and immunoassay (Ferri et al., 2009). In 2003, Sakurabayashi et al. (2003) described a new enzymatic assay involving the 
reaction of fructosyl amino acid oxidase (FAOX) with FV hydrolyzed from HbAc1 (Sakurabayashi et al., 2003).

FAOX is present in many microorganisms, including bacteria (Ferri, Sakaguchi, Goto, Tsugawa, \& Sode, 2005; Hirokawa \& Kajiyama, 2002), filamentous fungi (Akazawa, Karino, Yoshida, Katsuragi, \& Tani, 2004; Hirokawa, Gomi, Bakke, \& Kajiyama, 2003; Takahashi, Pischetsrieder, \& Monnier, 1997a; Yoshida et al., 1996; Yoshida, Sakai, Serata, Tani, \& Kato, 1995), and marine yeas (Sode, Ishimura, \& Tsugawa, 2001). Fungal FAOX exhibits activity toward $N^{\varepsilon}$-fructosyl-lysine ( $\varepsilon$ FLys) and $\mathrm{N}^{\varepsilon}$-fructosyl $\mathrm{N}^{\alpha}$-Z-lysine (Sakai, Yoshida, Isogai, Tani, \& Kato, 1995; Sakai, Yoshida, Tani, \& Kato, 1996; Takahashi et al., 1997a, Takahashi, Pischetsrieder, \& Monnier, 1997b; Wu, Takahashi, Chen, \& Monnier, 2000; Yoshida et al., 1995, 1996). The activity toward $\varepsilon F L y s$ interfere with that towards FV, resulting in inaccurate measurements of HbA1c using fungal FAOX. In 2003, Sakaue and Kajiyama discovered a FAOX from Corynebacterium sp. strain 2-4-1 (FAOX-C) that showed high activity toward FV, but no activity toward $\varepsilon$ FLysand $N^{\varepsilon}$-fructosyl $N^{\alpha}$-formyl-lysine (cFfLys) (Sakaue \& Kajiyama, 2003). They also engineered FOAX-C and obtained a variant called FOAX-TE that is significantly more thermal stable than FAOX-C (Sakaue \& Kajiyama, 2003). Additionally, FAOX-TE showed high specificity for FV and fructosyl glycine (FG) and no-detectable activity toward \&FLys (Sakaue \& Kajiyama, 2003). Thus, FAOX-TE is a promising enzyme for the further development of HbA1c quantification assays. In this study, we heterologously expressed in Escherichia coli and purified GST-tagged FAOX-TE (GST/FAOX-TE). The activity of the fusion protein was tested. The results obtained herein serves as the foundation for the further studies on developing biosensor for HbA1c using recombinant FAOX-TE.

\section{Materials and methods}

\section{Reagents}

BamHI-HF, XhoI, T4 DNA ligase and DNA polymerase were obtained from New England Biolabs (USA). Unstained Protein Ladder, Monarch ${ }^{\circledR}$ Plasmid Miniprep Kit and Monarch ${ }^{\circledR}$ DNA Gel Extraction Kit were also purchased from New England Biolabs (USA). Agar, peptone, yeast extract, sodium chloride, ampicillin and IPTG were obtained from Bioline (UK). All chemicals used in SDS-PAGE were purchased from Merck (Germany). Fructosyl valine (FV) was prepared as previously described (Keil, Mortensen, \& Christophersen, 1985; Rajkumar, Warsinke, Möhwald, Scheller, \& Katterle, 2007). Sodium hydroxide, sodium acetate, valine, and glucose were purchased from Sigma-Aldrich.

\section{Bacterial strains, media, Cell lines and vectors}

The E. coli strains $D H 5 \alpha$ and $B L 21$ (DE3) were used as cloning and expression hosts, respectively. The target DNA sequence coding for FAOX-TE was synthesized and inserted into the pUCIDT plasmid at IDT. The expression vector pGEX-4T3 was obtained from Cytiva (US).

\section{Construction of the vector for expressing GST-tagged FAOX-TE}

The pUCIDT-FAOX-TE (Figure 1) and pGEX-4T3 vectors were double-digested with the BamHI-HF and XhoI restriction enzymes following the manufacturer's instruction. The FAOXTE gene was inserted after the sequence of GST in the pGEX-4T3 vector, resulting in a fusion gene coding for GST-tagged FAOX-TE (Figure 2). The recombinant vector was transformed into the competent E. coli BL21 (DE3) by calcium chloride method (Yari, Afzali, Mozafari, Mansouri, \& Mostafaie, 2013). Transformants were selected on Lysogeny broth (LB) agar containing Ampicillin $(50 \mu \mathrm{g} / \mathrm{ml})$. Then, the colony PCR was applied to ensure the clones containing FAOX- 
TE gene using the forward primer pGEX-4T3-TAC (5'-AGCGGATAACAATTTCACACAGG3') and reverse primer pGEX-4T3-P3 (5'-CCGGGAGCTGCATGTGTCAGAGG-3'). The colonies containing FAOX-TE were subsequently cultured in LB liquid media containing Ampicillin $(50 \mu \mathrm{g} / \mathrm{ml})$, mixed with glycerol $\left(25 \% \mathrm{v} / \mathrm{v}\right.$ in final concentration) and stored at $-80^{\circ} \mathrm{C}$ for long-term usage.

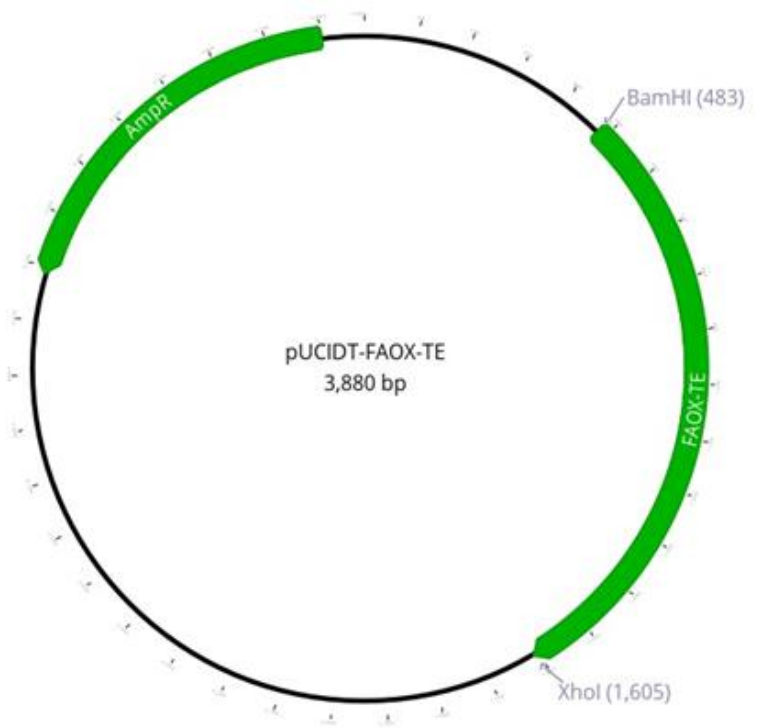

Figure 1. The pUCIDT-FAOX-TE vector

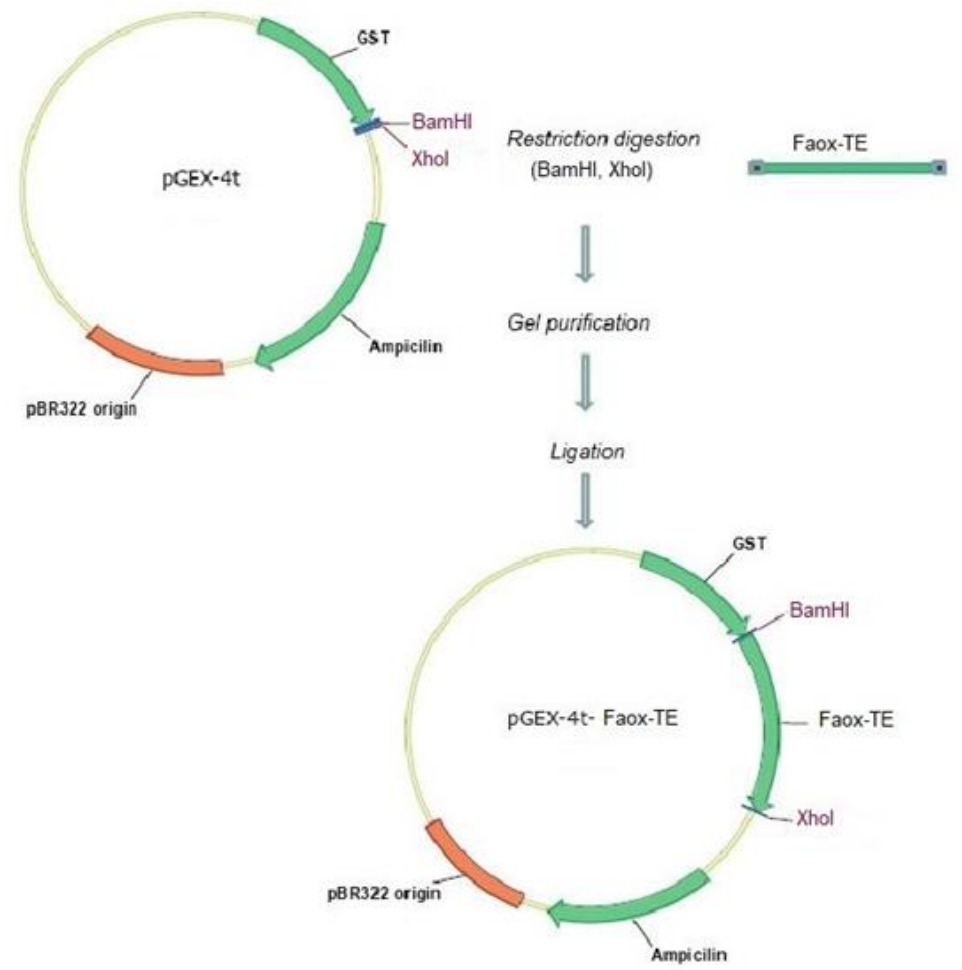

Figure 2. Schematic of the cloning procedure method applied to pGEX-4T3

Expression of GST/FAOX-TE

The frozen culture of E. coli BL21 (DE3) containing the plasmid pGEX-4T3-FAOX-TE was re-streaked on an LB agar plate, and several colonies were selected and inoculated with 
shaking ( $180 \mathrm{rpm})$ at $37^{\circ} \mathrm{C}$ in $10 \mathrm{ml}$ of LB liquid medium supplemented with ampicillin $(50 \mu \mathrm{g} / \mathrm{ml})$ overnight in a shaking incubator (Lab Companion, Korea). Then, $1 \mathrm{ml}$ of starter culture was transferred into $1 \mathrm{~L}$ Erlenmeyer flask containing $200 \mathrm{ml}$ of LB liquid medium and $50 \mu \mathrm{g} / \mathrm{ml}$ ampicillin, which was incubated in a shaker incubator at $37{ }^{\circ} \mathrm{C}$ and $180 \mathrm{rpm}$. When the OD of the culture reached 0.6 , the culture was induced with $0.5 \mathrm{mM}$ IPTG and subsequently incubated at 16 ${ }^{\circ} \mathrm{C}$ with shaking. The cells were harvested using centrifugation at $8,000 \mathrm{~g}$ for $15 \mathrm{~min}$ at $4{ }^{\circ} \mathrm{C}$.

\section{Purifications of GST/FAOX-TE}

The cell pellets (about $15 \mathrm{mg}$ ) were resuspended at a ratio of $1 \mathrm{mg}: 5 \mathrm{ml}$ of phosphate buffer pH 7.4 and sonicated (750 Watt Ultrasonic Processor, USA) at $20 \mathrm{kHz}$ frequency with $20 \%$ amplitude at alternating cycles of 5 seconds on and 10 second off. This equipment equipped with a $3 \mathrm{~mm}$ diameter titanium needle probe to disrupt the cells. The mixture was centrifuged at 16000 $\mathrm{g}$ for 15 minutes and the supernatant was collected as the total protein. The supernatant containing GST/FAOX-TE was loaded onto a column containing $2 \mathrm{ml}$ of Glutathione Sepharose 4 Fast Flow (Cytiva, US) at a flow rate of $1 \mathrm{ml} / \mathrm{min}$. The column was washed with $10 \mathrm{ml}$ of phosphate buffer $\mathrm{pH}$ 7.4. The bound GST/FAOX-TE was eluted with 3 fractions of Glutathione (20 mM). Final, PD MidiTrap G-25 was used for desalting and phosphate buffer exchange of GST/FAOX-TE samples.

\section{SDS-PAGE analysis}

The total protein sample and purified GST/FAOX-TE was checked using Sodium dodecyl sulphate polyacrylamide gel electrophoresis (SDS-PAGE). Briefly, about $15 \mu 1$ of samples were homogenized in SDS sample loading buffer. The total polyacrylamide concentration was $12 \%$ for separating gel and $4 \%$ for stacking gel. Protein bands were visualized using Coomassie Brilliant Blue R-250 (Sigma Andrich).

\section{Catalytic activity assay with Fructosyl Valine}

GST/FAOX-TE $(60 \mu \mathrm{M})$ was mixed with $3.8 \mathrm{mg} / \mathrm{mL}$ Fructosyl Valine (FV), and $100 \mathrm{mM}$ phosphate buffer $\mathrm{pH} 8.0$. The reaction mixture was incubated at $37{ }^{\circ} \mathrm{C}$ with rotation at $1000 \mathrm{rpm}$ for 10 minutes, followed by centrifugation at $16000 \mathrm{~g}$ for 10 minutes to eliminate debris. The solution was filtered through a $0.45 \mu \mathrm{m}$ membrane filter and then injected into the highperformance anion-exchange chromatography with Pulsed Amperometric Detection (HPAECPAD) system ( $25 \mu \mathrm{L}$ sample loop). The HPAEC-PAD DionexTM ICS 3000 system was equipped with a disposable electrochemical gold electrode and a CarboPac PA20 column (Dionex). An isocratic eluent with $90 \% 10 \mathrm{mM} \mathrm{NaOH}$ (eluent A) and $10 \% 250 \mathrm{mM} \mathrm{NaOH}$ (eluent B) was used to evaluate Valine at $30{ }^{\circ} \mathrm{C}$. The standard waveform for amino acids analysis was utilized. Data collection and analysis were carried out with the Chromeleon 6.8 software. In order to distinguish the products, FV (51ppm) and valine (42ppm) were also analyzed independently.

\section{Results and discussion}

\subsection{Obtaining FAOX-TE sequence}

The FAOX-TE gene was separated from the pUCIDT-FAOX-TE by double digestion with BamHI-HF and XhoI restriction enzymes. The FAOX-TE gene and pUCIDT fragment products appeared as $1100 \mathrm{bp}$ and $2700 \mathrm{bp}$ bands on $1 \%$ agarose gel, respectively (Figure 3). The FAOXTE gene was inserted into pGEX-4T3 vector and transformed into competent cells of E. coli DH5 $\alpha$ for propagation. As the result, E. coli $D H 5 \alpha$ strains containing recombinant plasmids were confirmed by colony PCR (data not shown). 


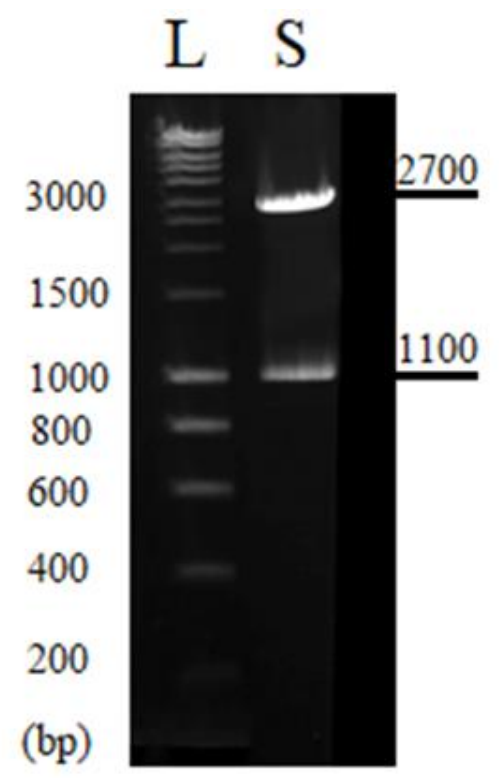

Figure 3. Electrophoresis analysis in $1 \%$ agarose gel of the plasmid pUCIDT-FAOX-TE digested with BamHI-HF and XhoI restriction enzymes. Lane L: Gene ruler DNA ladder; Lane S: FAOX gene (1100 bp) and pUCIDT fragment (2700 bp)

\subsection{Cloning of the pGEX-4T3-FAOX-TE expression vector}

The protein-coding sequence of the FAOX-TE gene was placed between the BamHI-HF and XhoI sites of the pGEX-4T3 vector, resulting in a fusion gene coding for a GST tag fused to the N-terminal of FAOX-TE. Competent E. coli BL21 (DE3) cells were transformed with the pGEX-4T3-vector. The transformed colonies were selected on LB agar containing ampicillin $(50 \mu \mathrm{g} / \mathrm{ml})$. The transformed colonies that contain pGEX-4T3-FAOX-TE plasmid were selected by colony PCR using FAOX-TE gene specific primers (data not shown). Subsequently, the recombinant plasmid was confirmed by double digestion with BamHI-HF and XhoI restriction enzymes. The FAOX-TE gene and pGEX-4T3 fragment appeared as 1100 bp and 5000 bp bands on $1 \%$ agarose gel, respectively (Figure 4 ).

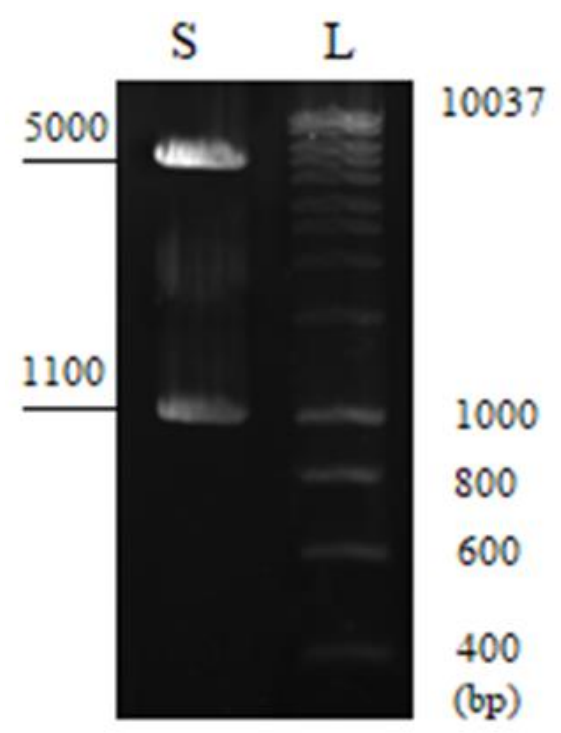

Figure 4. Electrophoresis analysis in 1\% agarose gel of pGEX-4T3-FAOX-TE digested with BamHI-HF and XhoI restriction enzymes. Lane S: FAOX gene (1100 bp) and pGEX-4T3 fragment (5000 bp); Lane L: Gene ruler DNA ladder 


\subsection{Expression of recombinant GST/FAOX-TE}

The total protein of induced non-transformed E. coli BL21 (DE3), as well as induced and non-induced transformed E. coli BL21 (DE3) cells were analyzed with SDS-PAGE (Figure 5). A band around $60-70 \mathrm{kDa}$ is highly enriched in the induced transformed cells but not visible in both the non-transformed cells and non-induced transformed cells. The estimated molecular weight of the protein in this band is consistent with the calculated molecular weight of GST/FAOX-TE.

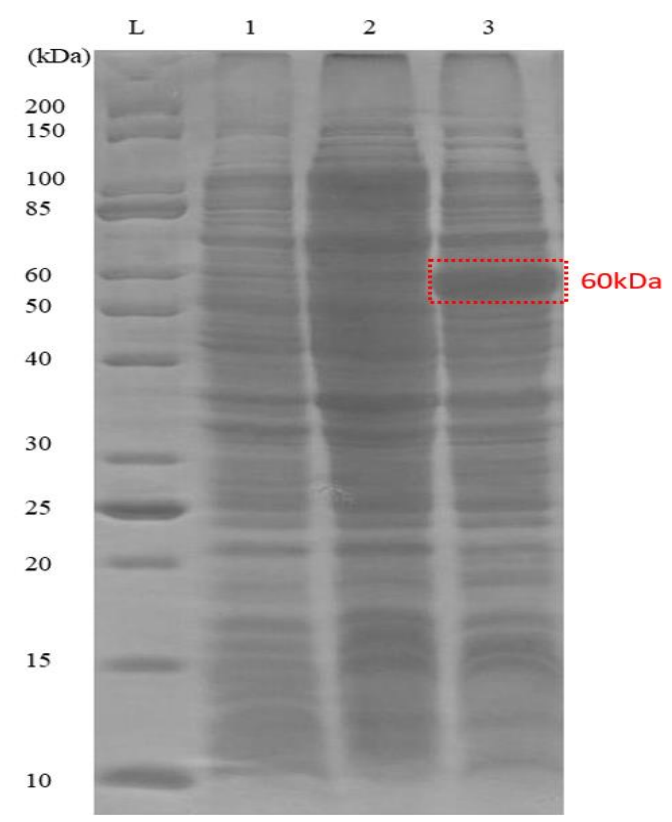

Figure 5. SDS-PAGE analysis of GST/FAOX-TE expression. Lane L: protein marker; Lane 1: total protein of non-transformed E. coli BL21 (DE3) induced with IPTG as a control; Lane 2: total protein of transformed cells without induction; Lane 3: total protein of transformed cells after induction with IPTG

\subsection{Purification of recombinant GST/FAOX-TE}

In order to purify GST/FAOX-TE, Glutathione Sepharose 4 Fast Flow (Cytiva, US) column was used. The recombinant GST/FAOX-TE fusion protein binds to the GST-tagged protein of Glutathione Sepharose 4 Fast Flow through the GST-tag. The target recombinant protein was eluted with 3 fractions by Glutathione $15 \mathrm{mM}$ (Figure 6, lane E1, E2 and E3). The purity of GST/FAOX-TE in fractions E1, E2, and E3 was estimated to be 85\%, 90\%, and 93\%, respectively.

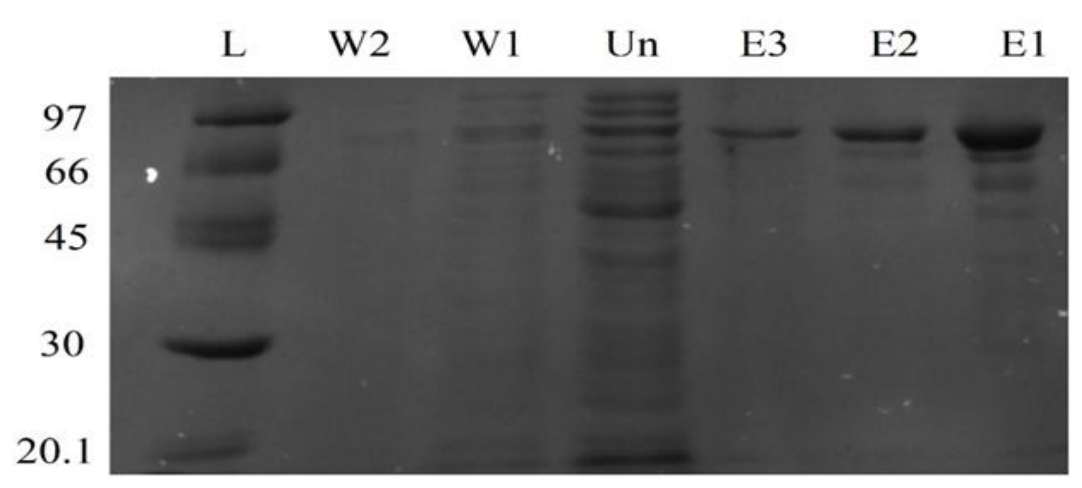

Figure 6. SDS-PAGE analysis of recombinant GST/FAOX-TE. Lane L: protein marker; Lane W1: First wash; Lane W2: Second wash; Lane Un: Flow through; Lanes E1, E2, and E3: three elution fractions of GST/FAOX-TE 


\subsection{Activity assay of GST/FAOX-TE with Fructosyl Valine (FV)}

FAOX oxidizes fructosyl valine, generating glucosone and valine (Sakaue \& Kajiyama, 2003) (Figure 7). The reaction products were analyzed with HPAEC-PAD (Figure 8). The HPAEC-PAD chromatogram of FV treated with GST/FAOX-TE exhibits a clear peak corresponding to valine $(6.8 \mathrm{~min})$. This peak is absent in both the chromatograms of untreated FV and the enzyme sample without FV. This result indicated that purified GST/FAOX-TE was active on fructosyl valine.

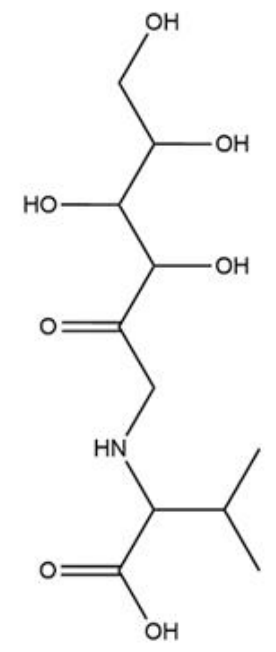

Fructosyl Valine

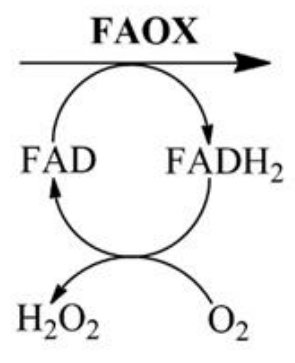

Glucosone

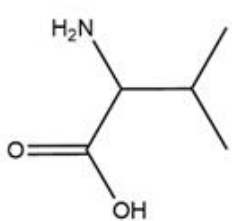

Valine

Figure 7. Fructosyl valine oxidation by FAOX

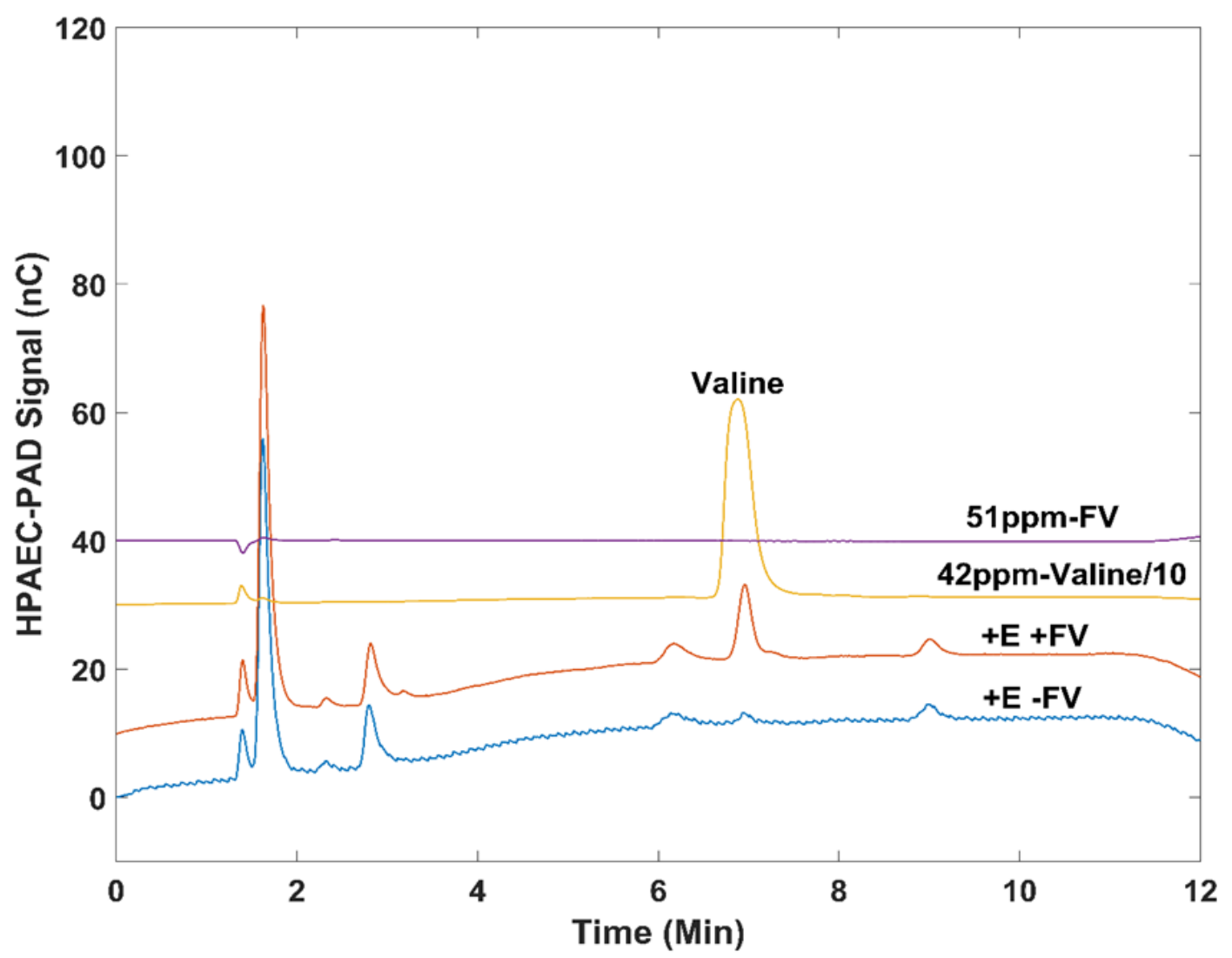

Figure 8. HPAEC-PAD analysis of FV $(3.8 \mathrm{mg} / \mathrm{mL})$ oxidation by GST/FAOX-TE $(60 \mu \mathrm{M})$ (noted as $\mathrm{E}$ in the traces) in $100 \mathrm{mM}$ phosphate buffer at $\mathrm{pH} 8.0$ 


\section{Conclusion}

GST/FAOX-TE was successfully expressed in E. coli BL21 (DE3) strain carrying the recombinant plasmid pGEX-4T3-FAOX-TE. The fusion protein was purified to more than $85 \%$ purity using the GST-tag. Initial assays indicated that the purified GST/FAOX-TE was active on FV. The results obtained in this work set the foundation for our further studies on developing biosensor for rapid FV quantification.

\section{ACKNOWLEDGMENTS}

This research is funded by NTTU Foundation for Science and Technology Development under grant number 2018.01.14.

\section{References}

Akazawa, S., Karino, T., Yoshida, N., Katsuragi, T., \& Tani, Y. (2004). Functional analysis of fructosyl-amino acid oxidases of aspergillus oryzae. Applied and Environmental Microbiology, 70(10), 5882-5890. doi:10.1128/AEM.70.10.5882-5890.2004

Bunn, H. F., Haney, D. N., Kamin, S., Gabbay, K. H., \& Gallop, P. M. (1976). The biosynthesis of human hemoglobin A1c. Slow glycosylation of hemoglobin in vivo. Journal of Clinical Investigation, 57(6), 1652-1659.

Ferri, S., Kim, S., Tsugawa, W., \& Sode, K. (2009). Review of fructosyl amino acid oxidase engineering research: A glimpse into the future of hemoglobin A1c biosensing. Journal of Diabetes Science and Technology, 3(3), 585-592. doi:10.1177/193229680900300324

Ferri, S., Sakaguchi, A., Goto, H., Tsugawa, W., \& Sode, K. (2005). Isolation and characterization of a fructosyl-amine oxidase from an arthrobacter sp. Biotechnology Letters, 27(1), 27-32. doi:10.1007/s10529-004-6312-Z

Hirokawa, K., Gomi, K., Bakke, M., \& Kajiyama, N. (2003). Distribution and properties of novel deglycating enzymes for fructosyl peptide in fungi. Archives of Microbiology, 180(3), 227231. doi:10.1007/s00203-003-0584-X

Hirokawa, K., \& Kajiyama, N. (2002). Recombinant agrobacterium AgaE-like protein with fructosyl amino acid oxidase activity. Bioscience, Biotechnology, and Biochemistry, 66(11), 2323-2329. doi:10.1271/bbb.66.2323

Keil, P., Mortensen, H. B., \& Christophersen, C. (1985). Fructosylvaline: A simple model of the N-terminal residue of human haemoglobin A1c. Acta Chemica Scandinavica. Series B: Organic Chemistry and Biochemistry, 39(3), 191-193. doi:10.3891/acta.chem.scand.39b0191

Rajkumar, R., Warsinke, A., Möhwald, H., Scheller, F. W., \& Katterle, M. (2007). Development of fructosyl valine binding polymers by covalent imprinting. Biosensors and Bioelectronics, 22(12), 3318-3325. doi:10.1016/j.bios.2007.03.001

Sakai, Y., Yoshida, N., Isogai, A., Tani, Y., \& Kato, N. (1995). Purification and properties of fructosyl lysine oxidase from Fusarium oxysporum S-1F4. Bioscience, Biotechnology, and Biochemistry, 59(3), 487-491. doi:10.1271/bbb.59.487

Sakai, Y., Yoshida, N., Tani, Y., \& Kato, N. (1996). Production of fructosyl lysine oxidase from Fusarium oxysporum S-1F4 on autoclave-browned medium. Bioscience, Biotechnology, and Biochemistry, 60(1), 150-151. doi:10.1271/bbb.60.150 
Sakaue, R., \& Kajiyama, N. (2003). Thermostabilization of bacterial fructosyl-amino acid oxidase by Directed evolution. Applied and Environmental Microbiology, 69(1), 139-145. doi:10.1128/AEM.69.1.139-145.2002

Sakurabayashi, I., Watano, T., Yonehara, S., Ishimaru, K., Hirai, K., Komori, T., \& Yagi, M. (2003). New enzymatic assay for glycohemoglobin. Clinical Chemistry, 49(2), 269-274. doi:10.1373/49.2.269

Sode, K., Ishimura, F., \& Tsugawa, W. (2001). Screening and characterization of fructosyl-valineutilizing marine microorganisms. Marine Biotechnology (New York, N.Y.), 3(2), 126-132. doi:10.1007/s101260000065

Takahashi, M., Pischetsrieder, M., \& Monnier, V. M. (1997a). Isolation, purification, and characterization of amadoriase isoenzymes (fructosyl amine-oxygen oxidoreductase EC 1.5.3) from Aspergillus sp. The Journal of Biological Chemistry, 272(6), 3437-3443. doi:10.1074/jbc.272.6.3437

Takahashi, M., Pischetsrieder, M., \& Monnier, V. M. (1997b). Molecular cloning and expression of amadoriase isoenzyme (fructosyl amine:oxygen oxidoreductase, EC 1.5.3) from Aspergillus fumigatus. The Journal of Biological Chemistry, 272(19), 12505-12507. doi:10.1074/jbc.272.19.12505

Wu, X., Takahashi, M., Chen, S. G., \& Monnier, V. M. (2000). Cloning of amadoriase I isoenzyme from Aspergillus sp: Evidence of FAD covalently linked to Cys342. Biochemistry, 39(6), 1515-1521. doi:10.1021/bi992031g

Yari, K., Afzali, S., Mozafari, H., Mansouri, K., \& Mostafaie, A. (2013). Molecular cloning, expression and purification of recombinant soluble mouse endostatin as an anti-angiogenic protein in Escherichia coli. Molecular Biology Reports, 40(2), 1027-1033. doi:10.1007/s11033-012-2144-4

Yoshida, N., Sakai, Y., Isogai, A., Fukuya, H., Yagi, M., Tani, Y., \& Kato, N. (1996). Primary structures of fungal fructosyl amino acid oxidases and their application to the measurement of glycated proteins. European Journal of Biochemistry, 242(3), 499-505. doi:10.1111/j.1432-1033.1996.0499r.x

Yoshida, N., Sakai, Y., Serata, M., Tani, Y., \& Kato, N. (1995). Distribution and properties of fructosyl amino acid oxidase in fungi. Applied and Environmental Microbiology, 61(12), 4487-4489. 